\title{
Complex Behavioral Strategy and Reversal Learning in the Water Maze without NMDA Receptor-Dependent Long-Term Potentiation
}

\author{
Tim Hoh, Jason Beiko, Francis Boon, Susannah Weiss, and Donald P. Cain \\ Department of Psychology and Graduate Program in Neuroscience, University of Western Ontario, \\ London, Ontario N6A 5C2, Canada
}

Successful performance of the water maze task requires that rats learn complex behavioral strategies for swimming in a pool of water, searching for and interacting with a hidden platform before its spatial location can be learned. To evaluate whether NMDA receptor-dependent long-term potentiation (NMDA-LTP) is required for learning the required behavioral strategies, rats with NMDA-LTP blocked by systemic pharmacological treatment were trained in the behavioral strategies using simplified and stepwise training methods. Despite the blockade of NMDA-LTP in the dentate gyrus and hippocampal area CA1,

The Morris water maze task is complex, requiring that rats learn various behavioral strategies (swim away from the pool wall; discover a hidden platform, which provides the only refuge; and mount and remain on the platform) before they can learn the specific location of the platform within the pool (Morris, 1989; Whishaw, 1989; Bannerman et al., 1995). Although some studies suggest that NMDA receptor-dependent long-term potentiation (NMDA-LTP) in the dentate gyrus is involved in place learning (Moser et al., 1998), others have shown that rats that are familiar with the required behavioral strategies are capable of learning the location of the platform without NMDA-LTP in the dentate gyrus (Bannerman et al., 1995; Saucier and Cain, 1995; Cain et al., 1997b). However, it is possible that learning the behavioral strategies themselves might involve hippocampal NMDA-LTP (Bliss and Collingridge, 1993; Bannerman et al., 1995; McHugh et al., 1996; Morris et al., 1996, 1997; Jeffrey, 1997). Naive rats given an NMDA receptor antagonist swim around the edge of the pool and often fail to mount and remain on the hidden platform when it is contacted (Whishaw and Auer, 1989; Saucier and Cain, 1995; Cain et al., 1996, 1997b; Saucier et al., 1996), suggesting that they are unable to learn the required strategies.

We evaluated whether NMDA-LTP is required for strategy learning by admnistering $[ \pm]$ cis-4-phosphono-methyl-2-piperidine carboxylic acid [CGS19755 (CGS)], a potent and specific NMDA receptor antagonist (Lehmann et al., 1988; Bennett et al., 1989). We took advantage of the fact that rats given simplified and stepwise training can perform the water maze task despite hippocampal formation damage or drug treatment known to severely impair performance in naive rats (Whishaw and Auer, 1989; Bannerman et al., 1995; Saucier and Cain, 1995; Whishaw et al.,

Received Dec. 1, 1998; revised Feb. 3, 1999; accepted Feb. 16, 1999.

This work was supported by a grant to D.P.C. from the Natural Science and Engineering Research Council of Canada. We thank Rob Sutherland for helpful suggestions about path integration.

Correspondence should be addressed to Prof. D. P. Cain, Department of Psychology, University of Western Ontario, London, Ontario N6A 5C2, Canada.

Copyright (C) 1999 Society for Neuroscience $0270-6474 / 99 / 190001-05 \$ 05.00 / 0$ rats learned the required behavioral strategies and used them to learn both initial and reversed platform locations. This is the first evaluation of the role of NMDA-LTP specifically in behavioral strategy learning. Although hippocampal NMDA-LTP might contribute to the water maze task, this form of LTP is not essential for learning complex behavioral strategies or multiple hidden platform locations.

Key words: water maze; nonspatial pretraining; LTP; hippocampus; spatial learning; strategy learning; NMDA
1995; Cain et al., 1996, 1997b; Day and Schallert, 1996; Saucier et al., 1996; Whishaw and Jarrard, 1996; Cain, 1997). Rats were first given different forms of simplified water maze pretraining, followed by conventional spatial training. CGS was administered before both phases of training to block NMDA-LTP. We expected that if learning the behavioral strategies depended critically on NMDA-LTP, the rats would not be able to learn the required behavioral strategies during pretraining and thus would not be able to learn the location of the hidden platform during subsequent spatial training (Morris, 1989; Whishaw, 1989; Whishaw et al., 1995; Whishaw and Jarrard, 1996). Electrophysiological experiments with subgroups of the same rats confirmed that CGS blocked NMDA-LTP in both the dentate gyrus and hippocampal area CA1. The role of NMDA-LTP in spatial reversal learning was also evaluated.

\section{MATERIALS AND METHODS}

\section{Behavioral procedures}

Behavioral training, pretrained groups. The drugged pretrained group $(n=$ 17) received nonspatial pretraining (NSP), a form of simplified training that allows rats to learn the required behavioral strategies but does not train them to find a specific platform location (Morris, 1989; Bannerman et al., 1995). Conventional NSP involves 12 spaced swims over $4 \mathrm{~d}$ (Morris, 1989). To avoid possible drug tolerance effects from repeated administration of CGS, we used a modified form of NSP that was given

This article is published in The Journal of Neuroscience, Rapid Communications Section, which publishes brief, peerreviewed papers online, not in print. Rapid Communications are posted online approximately one month earlier than they would appear if printed. They are listed in the Table of Contents of the next open issue of JNeurosci. Cite this article as: JNeurosci, 1999, 19:RC2 (1-5). The publication date is the date of posting online at www.jneurosci.org.

http://www.jneurosci.org/cgi/content/full/2973 
in one session (see below). Two days after NSP the rats were given a second injection of CGS before receiving 3 "reminder" NSP trials, followed immediately by spatial training. Thus all training was under CGS. A pretrained control group $(n=8)$ was given identical training but received saline instead of CGS. A naive drugged group $(n=12)$ received CGS and spatial training only.

A second pretrained group (visible pretrained group; $n=13$ ) was given simplified training based on the finding that training with a visible platform (Morris, 1984) can be used to pretrain rats in behavioral water maze strategies (Cain, 1997) or to train rats with hippocampal damage in both water maze strategies and acquisition of a place response (Whishaw et al., 1995). To avoid training a place response during this phase, the visible platform was moved to a new pool quadrant after every trial (Morris, 1984; Cain, 1997). The rats received CGS and training in the visible platform task, followed immediately by spatial training with a hidden platform in a fixed position, all in a single session. Control groups received an injection of saline followed by visible and hidden platform training, in that order $(n=9)$, or an injection of saline followed by hidden platform training alone $(n=7)$. These control groups did not differ on any measure in the hidden platform component $(p>0.05)$, presumably because of floor effects, and were combined to form the pooled control group.

Behavioral training, reversal group. Rats with hippocampal damage can learn a place response to an initial location when given simplified water maze training but are severely impaired in spatial reversal learning (Whishaw et al., 1995; Whishaw and Jarrard, 1996). To evaluate whether rats can learn both an initial place response and a spatial reversal (new fixed platform position) without NMDA-LTP, the reversal group $(n=$ 14) received conventional NSP (three trials per day, 4 d, no CGS; Morris, 1989) followed $5 \mathrm{~d}$ later by CGS and spatial training (hidden platform in southeast quadrant), followed $48 \mathrm{hr}$ later by CGS and spatial reversal training (hidden platform in northwest quadrant). CGS was administered only before the two spatial training sessions to avoid possible drug tolerance effects and because results from the drugged pretrained group indicated that CGS did not prevent robust strategy learning (see Results). A reversal control group $(n=8)$ was trained identically but received saline instead of CGS.

Apparatus and training protocols. The water maze was a circular pool, $1.5 \mathrm{~m}$ in diameter, filled with water at $29 \pm 1^{\circ} \mathrm{C}$, that was covered with floating polypropylene pellets and placed in a room with numerous distal cues. Heavy black curtains suspended on a ceiling-mounted track could be drawn around the water maze to occlude distal cues. Either a hidden or visible platform could be placed in the water maze $(15 \times 15 \mathrm{~cm}$; hidden, $1 \mathrm{~cm}$ below the surface; visible, $2 \mathrm{~cm}$ above the surface and marked with a 15 -cm-tall object). An overhead camera sent feed to a videocassette recorder and a computerized system (Poly-Track; San Diego Instruments, San Diego, CA) that digitized and objectively analyzed the swim paths for platform search time, time spent swimming in the periphery (the outer $50 \%$ of the pool, which never contained the platform), platform quadrant search time, and direct and circle swims (Saucier and Cain, 1995; Cain et al., 1996, 1997b; Whishaw and Jarrard, 1996). NSP for the drugged pretrained group consisted of 12 trials (60 sec maximum) in one session with black curtains around the water maze and the hidden platform moved to a new quadrant after every trial (Morris, 1989). Data from undrugged NSP control groups indicated that after NSP rats displayed significant place learning during subsequent spatial training (Morris, 1989; Saucier and Cain, 1995; Cain et al., 1997b) (see Fig. $2 a, d$ ), confirming that NSP did not train rats to perform a place response. Visible platform training for the visible pretrained group consisted of 10 trials in one session with the visible platform moved to a new quadrant after every trial. Spatial training consisted of 10 trials (60 sec maximum) in one session with no curtains and the hidden platform in the southeast quadrant. If a rat did not find the platform by $60 \mathrm{sec}$ it was placed on it by hand. Release points at the pool rim were from the cardinal compass points (north, south, east, and west) in random order. A probe trial consisting of a free swim $(60 \mathrm{sec})$ with the platform removed was given at the end of spatial training. To ensure that drugged groups were trained during the maximal effect of CGS, drugged and control groups were trained in squads of three with a $5 \mathrm{~min}$ intertrial interval, ensuring complete training within $1 \mathrm{hr}$ (Bennett et al., 1989). NSP for the reversal groups consisted of 12 trials (120 sec maximum, three trials per day, $4 \mathrm{hr}$ intertrial interval; Morris, 1989). Rats were placed under a heat lamp between trials. Rats with chronic electrode implants did not differ from rats without implants in the water maze task (Saucier and Cain, 1995), and induction or saturation of hippocampal
LTP did not affect water maze acquisition (Jeffrey and Morris, 1993; Korol et al., 1993; Saucier and Cain, 1995). Therefore we did not expect these treatments to affect the behavioral phase. Data were analyzed in blocks of two trials using repeated measures ANOVA and NeumannKeuls post hoc tests or $t$ tests (platform quadrant search time).

\section{LTP procedures}

Surgery. Male hooded rats were anesthetized (pentobarbital, $65 \mathrm{mg} / \mathrm{kg}$ ) and implanted with chronic stimulating and recording electrodes using conventional stereotaxic techniques under aseptic surgical conditions. Randomly selected rats from the drugged pretrained and visible pretrained groups received stimulating and recording electrodes in the perforant path and the ipsilateral dentate hilus (Saucier and Cain, 1995; Cain et al., 1997b) $(n=10)$ or a pair of recording electrodes that straddled the CA1 cell layer and a stimulating electrode in contralateral homotopic CA1 (Leung and Shen, 1993) $(n=10)$. A ground screw was placed in the skull. Final positioning of the electrodes during surgery was determined by monitoring field potentials evoked by single test pulses. Leads from the electrodes were connected to a plug attached to the skull with dental acrylic. The wound was closed, analgesics were administered, and the animal was given 1 week to recover. Electrode placements were confirmed using standard histological techniques. Procedures were performed in accordance with the guidelines of the Canadian Council on Animal Care.

Electrophysiological procedures. To evoke field potentials, single diphasic $(0.1 \mathrm{msec} / \mathrm{phase})$ test pulses were delivered to unanesthetized rats a minimum of $10 \mathrm{sec}$ apart. Evoked responses vary as a function of concurrent motor activity (Winson and Abzug, 1978; Leung, 1980; Moser et al., 1993). Therefore, all stimulation was delivered during behavioral immobility in a state of quiet wakefulness. Under our conditions this behavioral state is characterized by stable brain temperature and evoked field potential measures (Cain et al., 1994). Responses were digitized, averaged (10 sweeps), and analyzed for the rising phase of the field EPSP and the area under the evoked response (Leung and Shen, 1993; Saucier and Cain, 1995; Cain et al., 1997b). Input-output determination (five to seven test pulse intensities) was followed by CGS and redetermination of baseline $45 \mathrm{~min}$ later using a low-intensity test pulse suprathreshold for a dentate population spike or CA1 response. For dentate LTP, five high-frequency trains (50 diphasic pulses, $0.1 \mathrm{msec} /$ phase, $400 \mathrm{~Hz}$, nearmaximal input-output response) were applied to the perforant path (Cain et al., 1997b). For CA1 LTP, eight primed bursts (one pulse followed $190 \mathrm{msec}$ later by 10 pulses at $100 \mathrm{~Hz}$ ) at 1.2-2.0 times response threshold was used to avoid epileptiform afterdischarge (AD) (Leung and Shen, 1993). Electrographic activity at the recording site was continuously monitored to detect $\mathrm{AD}$; one rat with $\mathrm{AD}$ was excluded from the study. Averaged responses to the same test pulse were obtained 1 and $24 \mathrm{hr}$ later. To evaluate undrugged LTP, the procedure was repeated in the same rats, but CGS was not given. The order of testing of drugged (NMDA receptor antagonist) versus undrugged LTP induction did not affect the results in a counterbalanced study (Saucier and Cain, 1995). Therefore the order of testing was LTP attempted after injection of CGS, followed 1-2 weeks later by LTP attempted without CGS. Baseline measures did not differ between the CGS and no-treatment conditions $(p>0.05)$, indicating good stability of the experimental arrangements. The measures did not change $(p>0.05)$ after injection of CGS. Statistical comparisons between postdrug baseline and post-tetany values were made using the one-tailed Wilcoxon signed ranks test, because we predicted that under normal conditions the measures of LTP would increase (Bliss and Collingridge, 1993; Bannerman et al., 1995; Saucier and Cain, 1995).

Drug treatment. Published and dose-response pilot studies showed that CGS (4.0 mg/kg, i.p.) administered $45 \mathrm{~min}$ (Lehmann et al., 1988; Bennett et al., 1989) before tetany blocked dentate and CA1 LTP for $1 \mathrm{hr}$ (Cain et al., 1997b) and did not prevent rats from swimming effectively. Higher doses impaired swimming. Therefore $4.0 \mathrm{mg} / \mathrm{kg}$ CGS was administered $45 \mathrm{~min}$ before procedures requiring blockade of NMDA-LTP.

\section{RESULTS}

\section{Strategy learning during nonspatial pretraining}

Observation of the videotaped trials indicated that all of the pretrained groups learned to swim away from the wall to search for the platform in the inner $50 \%$ of the pool and to use the platform as a refuge when it was encountered. This was confirmed 
A

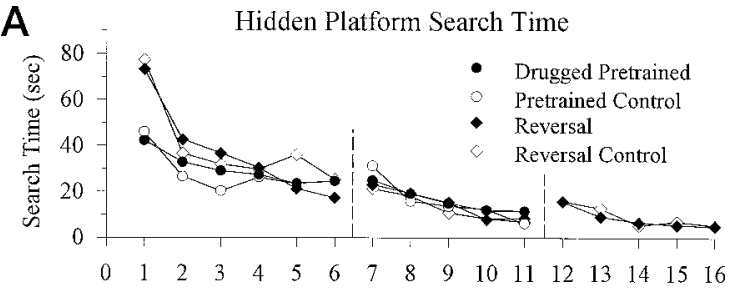

B
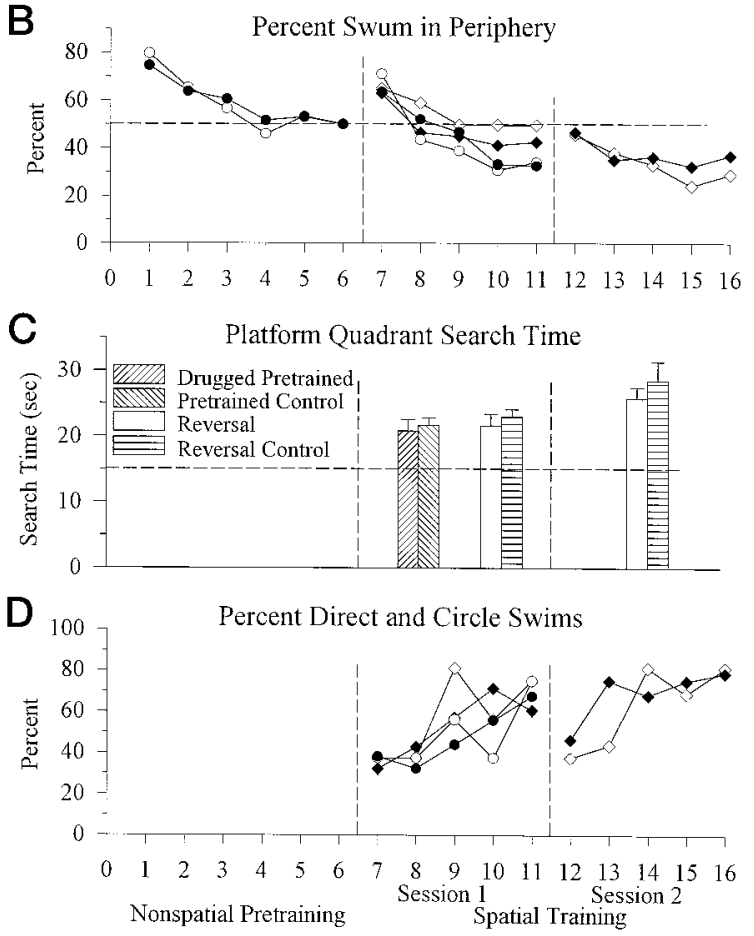

Figure 1. Water maze acquisition of drugged pretrained and reversal groups. $a$, Group mean platform search times (seconds) during nonspatial pretraining and spatial training plotted as two trials per block. $b$, Group mean percent of time swum in the pool periphery (the outer $50 \%$, which never contained a platform). Less than $50 \%$ swum in the periphery (below the dotted line) reflects acquisition of an effective search strategy focused on the inner part of the pool, which contained the hidden platform. $c$, Search time swum in the pool quadrant that contained the hidden platform during spatial training (dotted line, chance; $15 \mathrm{sec}$ ). $d$, Percent of swims during spatial training that were efficient direct or circle swims to the hidden platform (Whishaw et al., 1995; Whishaw and Jarrard, 1996).

by the absence of group and interaction effects for the drugged pretrained and pretrained control groups (search time, $p>0.05$; percent swum in the periphery, $p>0.05)$ and by significant decreases in search time (trial blocks, $F_{(5,115)}=6.7 ; p<0.0001$ ) and percent swum in the periphery (trial blocks, $F_{(5,115)}=11.0$; $p<0.0001$; Fig. $1 a, b)$ as pretraining progressed.

Observation of the videotaped trials similarly indicated that the visible pretrained group learned to use the visible platform as a refuge, demonstrating decreases in platform search time as training progressed (trial blocks, $F_{(4,28)}=3.7 ; p<0.02$; Fig. $2 a$ ).

\section{Spatial learning}

During spatial training, platform search time and periphery swimming declined further for the drugged pretrained and pretrained control groups (search time: groups, $p>0.05$; trial blocks, $F_{(4,92)}=12.5 ; p<0.0001$; interaction, $p>0.05$; percent swum in the periphery: groups, $p>0.05$; trial blocks, $F_{(4,92)}=18.2 ; p<$ 0.0001 ; interaction, $p>0.05$; Fig. $1 a, b)$. Additional measures
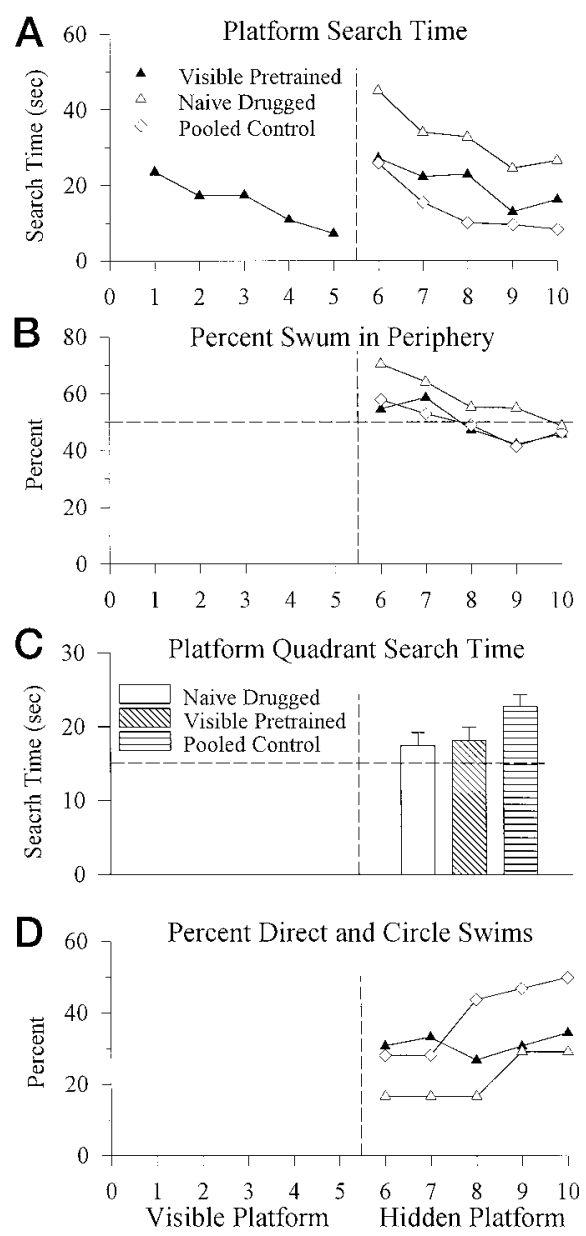

Figure 2. Water maze acquisition of the visible pretrained group. $a$, Group mean platform search times (seconds) during visible platform pretraining and spatial training plotted as two trials per block. $b$, Group mean percent of time swum in the pool periphery (see Fig. 1b). $c$, Search time swum in the pool quadrant that contained the hidden platform during spatial training (see Fig. 1c). $d$, Percent of swims during spatial training that were efficient direct or circle swims to the hidden platform.

confirmed that both groups made use of the behavioral strategies to learn the location of the hidden platform [platform quadrant search time, each group vs chance (15 sec), $p<0.002$; percent direct and circle swims, groups, $p>0.05$; trial blocks, $F_{(4,92)}=$ 4.2; $p<0.004$; interaction, $p>0.05$; Fig. $1 c, d)$. Formal evaluation of the rats' ability to make use of the platform as a refuge indicated that during the last half of NSP and throughout spatial training the groups did not differ in platform contact errors (failure to mount the platform when it was contacted) (Cain et al., 1996; data not shown). Taken together the measures indicated that the drugged pretrained group learned the behavioral water maze strategies during NSP and made use of them to learn the location of the hidden platform during spatial training.

Results for the visible pretrained and related control groups were similar. Hidden platform search time did not differ between the visible pretrained and pooled control groups (groups, $\mathrm{F}_{(2,8)}=$ $7.9 ; p<0.002$; trial blocks, $F_{(8,152)}=15.3 ; p<0.0001$; interaction, $p>0.05$; visible pretrained vs pooled control, $p>0.05$; visible pretrained and pooled control vs naive drugged, each $p<0.05$; Fig. 2a). The percent of time spent swimming in the periphery declined and did not differ between groups (groups, $p>0.05$; trial 

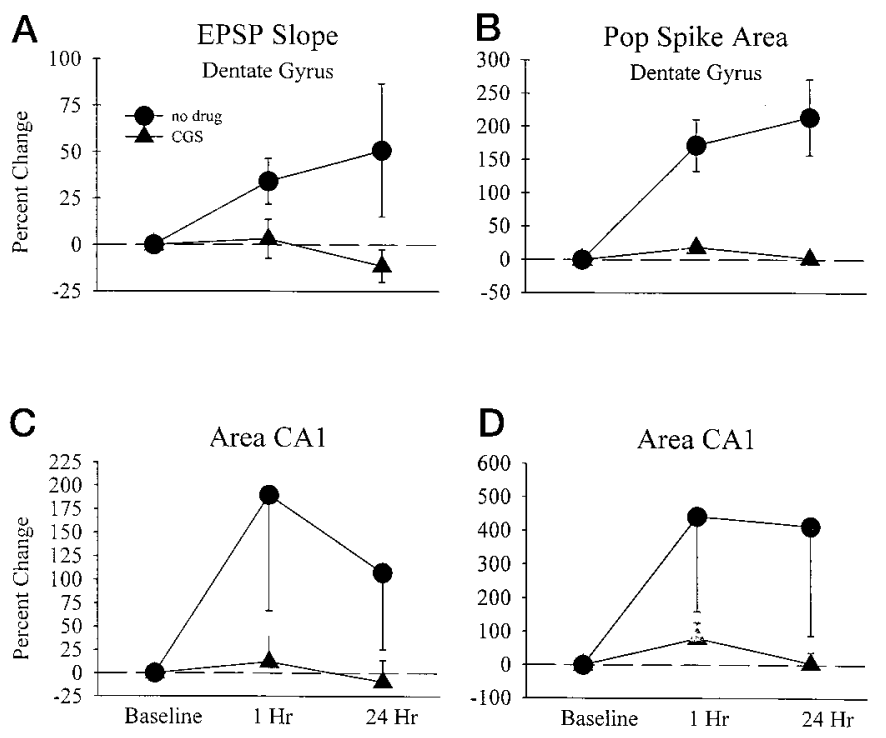

Figure 3. LTP and blockade of LTP by CGS in rats from the drugged pretrained and visible pretrained groups. $a, b$, Group mean percent of the baseline dentate gyrus field potential EPSP slope and population spike area (Pop Spike) at 1 and 24 hr after tetany when rats were pretreated with CGS or given no treatment (no drug). c,d, Group mean percent of the baseline hippocampal area CA1 field potential EPSP slope and population spike area at 1 and $24 \mathrm{hr}$ after tetany when rats were pretreated with CGS or given no treatment. CGS blocked LTP, but tetany induced significant LTP in the same rats 1-2 weeks later when no CGS was administered. Error bars indicate SEM.

blocks, $F_{(4,152)}=11.2 ; p<0.0001$; interaction, $p>0.05$; Fig. $\left.2 b\right)$. Additional measures confirmed that the naive drugged group were impaired, but the visible pretrained and pooled control groups learned the location of the hidden platform (platform quadrant search time, visible pretrained, pooled control, and naive drugged vs chance: $p<0.05, p<0.002$, and $p>0.05$, respectively; percent direct and circle swims: groups, $F_{(2,8)}=3.8$; $p<0.04$; trial blocks, $F_{(8,152)}=4.2 ; p<0.003$; interaction, $p>$ 0.05 ; pooled control vs naive drugged, $p<0.05$; Fig. $2 c, d$ ). Evaluation of the rats' ability to make use of the platform as a refuge indicated that the visible pretrained and pooled control groups did not differ in platform contact errors, and that both groups had fewer errors than the naive drugged group (data not shown). Although the visible pretrained group appeared to perform somewhat less effectively than the drugged pretrained group (Fig. 1), presumably because the visible pretrained group received fewer training trials presented in massed format, the visible pretrained group nevertheless showed clear evidence of learning both the behavioral strategies during visible platform training and the location of the hidden platform during subsequent spatial training.

\section{Spatial reversal learning}

The reversal group acquired both the initial and reversal place responses rapidly and did not differ from the reversal control group (all measures, $p>0.05$ vs reversal control; Fig. $1 a-d$ ).

\section{NMDA LTP blockade}

CGS blocked LTP in both the dentate gyrus and CA1 (all $p>$ 0.05 ; Fig. $3 a-d$ ). In the absence of CGS, high-frequency trains induced LTP in the same animals (all $p<0.05$; Fig. $3 a-d$ ). In both NSP experiments the behavioral results were comparable when rats that did not have blockade of NMDA-LTP by CGS evaluated electrophysiologically were excluded from the statistical analyses.

\section{DISCUSSION}

Previous research has shown that rats are capable of learning a place response in the water maze without NMDA-LTP if they are already familiar with the behavioral strategies required for this task (Bannerman et al., 1995; Saucier and Cain, 1995; Cain et al., 1996; Saucier et al., 1996). Our results show that learning the behavioral strategies themselves can occur without hippocampal NMDA-LTP, and that rats can then use the strategies to learn a place response. These findings are important because strategy learning is a complex form of cognitive learning that appears to involve associating sequences of behavior with outcomes. For example, thigmotaxic swimming around the periphery of the pool, which is common in naive rats given a variety of drug treatments (Whishaw and Auer, 1989; Paylor and Rudy, 1990; Saucier and Cain, 1995; Cain et al., 1996; Saucier et al., 1996; Cain, 1997), involves sequential swimming movements that do not have an adaptive outcome. In contrast, if the rat uses turning and swimming movements to direct itself away from the wall to the inner $50 \%$ of the pool where the hidden platform is located, the movements do have an adaptive outcome. Similarly, contact with the hidden platform followed immediately by swimming movements (Cain et al., 1996) do not have an adaptive outcome, whereas contact with the platform followed immediately by climbing movements do have an adaptive outcome.

Our findings are in stark contrast to results from many studies in which naive rats given NMDA receptor antagonists were severely impaired in the water maze task. Our finding of rapid spatial reversal learning in NSP rats given CGS also contrasts with the severe spatial reversal learning impairment seen in naive rats with hippocampal damage (Whishaw et al., 1995; Whishaw and Jarrard, 1996). Because not all LTP requires NMDA receptor activity (Harris and Cotman, 1986), it would be of interest to determine whether the present findings would generalize to other forms of LTP.

\section{The role of task strategies and task difficulty}

These and other data indicate that knowledge of the required task strategies plays an essential role in task acquisition and performance (Lashley, 1950; Morris, 1989; Whishaw, 1989). Separating water maze task components into behavioral strategy learning and place learning may facilitate task acquisition by reducing task difficulty at each stage in the training, discouraging the learning of counterproductive behaviors (Day and Schallert, 1996), or both. Naive rats given drug treatments may be impaired in their acquisition of the water maze task because they are required to learn both components of the task simultaneously.

This suggestion is consistent with the finding that an apparently more difficult version of the water maze task that involved a larger pool and smaller platform than were used here, with delayed matching-to-place training protocols (repeated reversal learning of platform position during $9 \mathrm{~d}$ ), yielded a place-learning impairment in NSP rats given spatial training under the NMDA receptor antagonist D-AP-5 (Morris et al., 1997). Thus, although less difficult place-learning tasks do not appear to require NMDALTP, more difficult place-learning tasks may require this form of LTP.

This effect of task difficulty may be related to an ability to use path integration (Barlow, 1964; McNaughton et al., 1996) for easy but not difficult place-learning tasks. Because path integration 
may play an important role in facilitating place learning (Cain et al., 1997a; Whishaw et al., 1997), it is possible that the pretraining procedures used here, which involve self-propelled exploration in the task environment, may produce their beneficial effects by enabling rats to acquire both general water maze task strategies and the ability to integrate self-generated motion as a means of navigation.

\section{REFERENCES}

Bannerman DM, Good MA, Butcher SP, Ramsay M, Morris RGM (1995) Distinct components of spatial learning revealed by prior training and NMDA receptor blockade. Nature 378:182-186.

Barlow JS (1964) Inertial navigation as a basis for animal navigation. J Theor Biol 6:76-117.

Bennett DA, Bernard PS, Amrick CL, Wilson DE, Liebman JM, Hutchison AJ (1989) Behavioral pharmacological profile of CGS19755, a competitive antagonist at $N$-methyl-D-aspartate receptors. J Pharmacol Exp Ther 250:454-460.

Bliss TVP, Collingridge GL (1993) A synaptic model of memory: longterm potentiation in the hippocampus. Nature 361:31-39.

Cain DP (1997) Prior non-spatial pretraining eliminates sensorimotor disturbances and impairments in water maze learning caused by diazepam. Psychopharmacology 130:313-319.

Cain DP, Hargreaves EL, Boon F (1994) Brain temperature- and behavior-related changes in the dentate gyrus field potential during sleep, cold water immersion, radiant heating, and urethane anesthesia. Brain Res 658:135-144.

Cain DP, Saucier D, Hall J, Hargreaves EL, Boon F (1996) Detailed behavioral analysis of water maze acquisition under APV or CNQX: contribution of sensorimotor disturbances to drug-induced acquisition deficits. Behav Neurosci 110:86-102.

Cain DP, Beiko J, Boon F (1997a) Navigation in the water maze: the role of proximal and distal visual cues, path integration, and magnetic field information. Psychobiology 25:286-293.

Cain DP, Saucier D, Boon F (1997b) Testing hypotheses of spatial learning: the role of NMDA receptors and NMDA-mediated long-term potentiation. Behav Brain Res 84:179-193.

Day LB, Schallert T (1996) Anticholinergic effects on acquisition of place learning in the Morris water task: spatial mapping deficit or inability to inhibit non-place strategies? Behav Neurosci 110:998-1005.

Harris EW, Cotman CW (1986) Long-term potentiation of guinea pig mossy fiber responses is not blocked by $\mathrm{N}$-methyl $\mathrm{D}$-aspartate antagonists. Neurosci Lett 70:132-137.

Jeffery KJ (1997) LTP and spatial learning-where to next? Hippocampus 7:95-110.

Jeffery K, Morris RGM (1993) Cumulative long-term potentiation in the rat dentate gyrus correlates with, but does not modify, performance in the water maze. Hippocampus 3:133-140.

Korol D, Abel T, Church L, Barnes C, McNaughton B (1993) Hippocampal synaptic enhancement and spatial learning in the Morris swim task. Hippocampus 3:127-132.

Lashley KS (1950) In search of the engram. Symp Soc Exp Biol $4: 454-482$

Lehmann J, Hutchison AJ, McPherson SE, Mondadore C, Schmutz M, Sinton CM, Tsai C, Murphy DE, Steel DJ, Williams M, Cheny DL, Wood PL (1988) CGS19755, a selective and competitive $N$-methyl-Daspartate-type excitatory amino acid antagonist. J Pharmacol Exp Ther 246:65-75.
Leung LS (1980) Behavior-dependent evoked potentials in the hippocampal CA1 region of the rat. I. Correlation with behavior and EEG. Brain Res 198:95-117.

Leung LS, Shen B (1993) Long-term potentiation in hippocampal CA1: effects of afterdischarges, NMDA antagonists, and anticonvulsants. Exp Neurol 119:205-214.

McHugh TJ, Blum KL, Tsien J, Tonegawa S, Wilson M (1996) Impaired hippocampal representation of space in CA1-specific NMDAR12 knockout mice. Cell 87:1339-1349.

McNaughton BL, Barnes CA, Gerrard JL, Gothard K, Jung MW, Knierm JJ, Kudrimoti H, Quin Y, Skaggs WE, Suster M, Weaver KL (1996) Deciphering the hippocampal polyglot: the hippocampus as a path integration system. J Exp Biol 199:173-185.

Morris RGM (1984) Developments of a water-maze procedure for studying spatial learning in the rat. J Neurosci Methods 11:47-60.

Morris RGM (1989) Synaptic plasticity and learning: selective impairment of learning in rats and blockade of long-term potentiation in vivo by the $N$-methyl-D-aspartate receptor antagonist AP5. J Neurosci 9:3040-3057.

Morris RGM, Bannerman DM, Good MA (1996) NMDA receptors and spatial learning: differential pretraining dissociates the behavioral effects of the receptor antagonist AP5 and selective hippocampal lesions. In: The hippocampus: functions and clinical relevance (Kato N, ed), pp 55-73. New York: Elsevier.

Morris RGM, Steele R, Martin SJ, Bell JE (1997) Hippocampal NMDA receptor function: role in memory or sensorimotor function? Soc Neurosci Abstr 23:217.

Moser E, Mathiesen I, Andersen P (1993) Association between brain temperature and dentate field potentials in exploring and swimming rats. Science 259:1324-1326.

Moser E, Krobert KA, Moser M-B, Morris RGM (1998) Impaired spatial learning after saturation of long-term potentiation. Science 281:2038-2042.

Paylor R, Rudy JW (1990) Cholinergic receptor blockade can impair the rat's performance on both the place learning and cued versions of the Morris water task: the role of age and pool wall brightness. Behav Brain Res 36:79-90.

Saucier D, Cain DP (1995) Spatial learning without NMDA receptordependent long-term potentiation. Nature 378:186-189.

Saucier D, Hargreaves EL, Boon F, Vanderwolf CH, Cain DP (1996) Detailed behavioral analysis of water maze acquisition under systemic NMDA or muscarinic antagonism: nonspatial pretraining eliminates spatial learning deficits. Behav Neurosci 110:103-116.

Whishaw IQ (1989) Dissociating performance and learning deficits on spatial navigation tasks in rats subjected to cholinergic muscarinic blockade. Brain Res Bull 23:347-358.

Whishaw IQ, Auer N (1989) Immediate and long-lasting effects of MK801 on motor activity, spatial navigation in a swimming pool and EEG in the rat. Psychopharmacology 98:500-507.

Whishaw IQ, Jarrard LE (1996) Evidence for extrahippocampal involvement in place learning and hippocampal involvement in path integration. Hippocampus 6:513-524.

Whishaw IQ, Cassel J-C, Jarrard LE (1995) Rats with fimbria-fornix lesions display a place response in a swimming pool: a dissociation between getting there and knowing where. J Neurosci 15:5779-5788.

Whishaw IQ, McKenna JE, Maaswinkel H (1997) Hippocampal lesions and path integration. Curr Opin Neurobiol 7:228-234.

Winson J, Abzug C (1978) Neuronal transmission through hippocampal pathways dependent on behavior. J Neurophysiol 41:716-732. 